\title{
Some Monte Carlo Experiments in Computing Multiple Integrals
}

1. Introduction. Despite the large volume of theoretical material on Monte Carlo methods which has appeared in the last five years, relatively little has been published on the experimental side of this technique [1]. The numerical analyst who is confronted with production computation is frequently anxious to obtain the "feel" of the method by looking at the results of the technique when applied to simple problems. It is towards this end that we present here the results of computing the volumes of $n$-dimensional hyperspheres $(n=2,3, \cdots, 12)$ by Monte Carlo methods. These computations were carried out on the National Bureau of Standards Eastern Automatic Computer (SEAC).

2. The Problem Solved: An $n$-dimensional hypersphere $H_{n}$ is defined by the inequality

$$
x_{1}{ }^{2}+x_{2}{ }^{2}+\cdots+x_{n}{ }^{2} \leq 1
$$

Its volume $V_{n}$ is given by

$$
V_{n}=\frac{2}{n} \frac{\pi^{n / 2}}{\Gamma(n / 2)}
$$

if $E_{n}$ designates the hypercube

$$
-1 \leq x_{i} \leq 1 \quad(i=1,2, \cdots, n)
$$

then the fraction of the volume of $E_{n}$ which is contained in $H_{n}$ is

$$
v_{n}=\frac{\pi^{n / 2}}{n 2^{n-1} \Gamma(n / 2)}
$$

The quantity $v_{n}$ will be called the normalized volume. The ratio $v_{n}$ may be computed in Monte Carlo style by generating a pseudo-random sequence of points $P_{1}, P_{2}, \cdots$ which lie in $E_{n}$ and finding the success ratio, i.e., the fraction of the points which lie in $H_{n}$. In the computations carried out, no use was made of the symmetries of $H_{n}$, nor was any attempt made to carry out any of the ideas of "importance sampling" [2].

3. Generation of Pseudo-Random Sequences: Two methods were employed to generate a pseudo-random sequence [3]. Method $I$ is that of successive low order multiplication by $5^{17} \cdot 2^{-42}$. This can be described by

$$
\begin{aligned}
u_{j+1} & =2^{-1 v_{v}} \\
v_{j+1} & =5^{17} v_{j}\left(\bmod 2^{42}\right), \quad v_{0}=1 .
\end{aligned}
$$

Method II is one which uses modified Fibonacci sequences. Starting from two 
arbitrarily given values $u_{0}, u_{1} \leq 4$, we form successively

$$
u_{j+1}=u_{j}+u_{j-1} \bmod 4 .
$$

The interest in the second method lies in its utilization of addition as opposed to multiplication and raises the possibility of consuming less time. Some preliminary statistical work on these sequences [4] indicated that it was better to select every second number of the sequence $(6)$. With the aim of scrambling the fundamental sequence (6) even further, we introduced skips of length 1, 2, 3 in (6) depending upon whether the last hexidecimal digit of the last number selected was $=1,2$, or 0 , mod 3. In the runs which were tabulated under Method II, the starting values $u_{0}=0$ and $u_{1}=2^{-42}$ were selected. In the runs tabulated under Method $\mathrm{II}^{\prime}$, the starting values $u_{0}=\pi, u_{1}=5^{17} \cdot 2^{-42}$ were used.

To generate a sequence of points $P_{1}, P_{2}, \cdots$, in $E_{n}$, the following scheme was adopted. The fundamental sequence was normalized so that $-1 \leq u_{j} \leq 1$. The computing machine then inquired: is $u_{1}{ }^{2}+u_{2}{ }^{2} \leq 1$ ? If so, is $u_{1}{ }^{2}+u_{2}{ }^{2}+u_{3}{ }^{3} \leq 1$ ?, $\cdots$, etc. If all these questions were answered affirmatively, then $P_{1}:\left(u_{1}, \cdots, u_{n}\right)$ and $P_{1} \in H_{n}$. If the question was answered negatively at some point, say $u_{1}{ }^{2}+u_{2}{ }^{2}+\cdots+u_{p}{ }^{2}>1, p \leq n$, then $u_{1}, \cdots, u_{p}$ were considered as the first $p$ coordinates of a point $P_{1}$ not in $H_{n}$. The remaining coordinates $u_{p+1}, \cdots, u_{n}$ were then not discarded, but the same procedure was repeated for $P_{2}$ beginning with $u_{p+1}$. That is, if $P_{1} \in H_{n}$ then $P_{2}:\left(u_{n+1}, u_{n+2}, \cdots, u_{n+p^{\prime}}, \cdots\right)$, but if $P_{1}$ is not in $H_{n}$, then $P_{2}$ has its first coordinate beginning with $u_{p+1}$.

4. Quality of Results : According to statistical theory [5], the probable error (error at the $50 \%$ level) in a computation with $N$ points is

$$
e_{50}=.6745 \sqrt{\frac{v_{n}\left(1-v_{n}\right)}{N}}
$$

while the error at the $95 \%$ level is given by

$$
c_{95}=1.96 \sqrt{\frac{v_{n}\left(1-v_{n}\right)}{N}} .
$$

With Method I, the observed error was less than $e_{95}$ in 10 of the twelve cases and exceeded it by about $20 \%$ in two of the twelve cases. The quality of Method II is somewhat dubious. The error estimate $e_{95}$ was exceeded in two cases out of six, and in the case $n=9$, the discrepancy is pronounced. Convergence seems to be taking place towards the wrong value. Method II' (which differs from Method II only in the starting values) seems to be considerably better and on a par with Method I. In view of the erratic nature of the results of Method II and its apparent sensitivity to starting values, we do not recommend that it be used for purposes of multiple integration. Method I seems to be satisfactory and the results are in conformity with the $1 / \sqrt{N}$ law for accuracy. This law is extremely conservative and precludes many computations from being carried out economically, and if, indeed, the present speed of electronic calculators were increased by, say, a factor of 100 this would result in only one more decimal place of accuracy for a fixed amount of computing time. As it is, using Method I, the approximate 
TABLE I

Computation of Normalized Volume of $n$-Dimensional Hiypersphere by Sampling

No. of Method I

Points

Sampled $\quad n=2$

$n=3$

32

0.8750000000

.8281250000

.7812500000

.7929687500

.7851562500

.8027343750

.7880859375

.7817382813

.7860107422

.7907714844

.7890014648
0.5000000000

.5000000000

.5468750000

.5625000000

.5449218750

.5341796875

.5307617188

.5219726563

.5224609375

.5299682617

.5272216797

$$
n=4
$$

0.2812500000

.3125000000

.3046875000

.3242187500

.3398437500

.3183593750

.3144531250

.3022460938

.3067626953

.3120117188

.3111267090

$$
n=5
$$

0.2187500000 .2187500000 .2187500000 .1835937500 .2011718750 .1757812500 .1708984375 .1638183594 .1647949219 .1635742188 .1646423340

Exact
Answer
Error
Predicted

0.785398

0.523599

0.308425

0.164493

0.003603

0.003623

0.002702

0.000149

Error at

95\% Level 0.004445

0.005408

0.005001

0.004014

No. of

Points

Sampled

$$
n=6
$$

$$
n=7
$$

$$
n=8
$$

$$
n=9
$$

0.0000000000

0.0000000000

0.0000000000

.0312500000

.0156250000

.0156250000

$.0390625000 \quad .0234375000$

.0468750000

.0273437500

.0312500000

.0156250000

.0449218750

.0361328125

.0917968750

1024

2048

4096

8192

16384

32768

.0825195313

.0778808594

.0822753906

.0800170898

65536

.0317382813

.0319824219

.0346679688

.0342407227

.0792236328

.0344543457

.0195312500

.0156250000

.0151367188

.0144042969

.0136108398

.0142517090

.0214843750

.0126953125

.0087890625

.0078125000

.0065917969

.0056762695

.0057983398

.0061340332

Exact

Answer

0.080746

0.036912

0.015854

0.006442

Error

0.001522

0.002458

0.001602

0.000308

Predicted

Error at

$95 \%$ Level 0.002950

0.002041

0.001352

0.000612 
TABLE I-Continued

No. of

Points

Sampled

$$
n=10
$$

$n=11$

$n=12$

$\begin{array}{rr}32 & 0.0000000000 \\ 64 & .0000000000 \\ 128 & .0078125000 \\ 256 & .0039062500 \\ 512 & .0078125000 \\ 1024 & .0048828125 \\ 2048 & .0024414063 \\ 4096 & .0024414063 \\ 8192 & .0021972656 \\ 16384 & .0015869141 \\ 32768 & .0017700195 \\ 65536 & .0022277832\end{array}$

Exact

Answer

0.002490

Error

0.000262

Predicted

Error at

95\% Level 0.000540

Number

of Points

Sampled

$$
n=2
$$

0.125

.46875

.65625

.7421875

.7695313

.7851563

.7783203

.7792969

.7861328

.7784424

.7832642

.7859802

.7859192
0.0000000000 .0000000000 .0078125000 .0039062500 .0039062500 .0019531250 .0009765625 .0009765625 .0007324219 .0006713867 .0007934570 .0010223389

0.000920

0.000102

0.0000000000 .0000000000 .0000000000 .0000000000 .0019531250 .0009765625 .0004882813 .0002441406 .0001220703 .0002441406 .0003051758 .0004425049
\4096

16384

32768

65536

0.000232

0.000138
Method II

$$
n=3
$$

0.125

.3125

.453125

.5000000

.5390625

.5214844

.5380859

.5327148

\subsection{6}

0.000117
.31323242

Exact

Answer

0.785398

0.5235987

0.308425

0.164493

Error

$$
0.000521
$$

0.0091

0.004807

0.0051

Predicted

Error at

95\% Level

0.003143

0.021631

0.010001

0.011353

0.000

.03125

.109375

.1171875

.1406250

.1503906

.1572266

.1645507

.1594238 


\section{TABLE I-Continued}

Number

of Points

Sampled

$$
n=6
$$

$$
n=9
$$

16
32
64
128
256
512
1024
2048
4096
8192
16384
32768

$$
\begin{aligned}
& 0.000 \\
& .03125 \\
& .078125 \\
& .0859375 \\
& .0742188 \\
& .0761719 \\
& .0791016 \\
& .0786133 \\
& .0764160
\end{aligned}
$$$$
0.000
$$$$
.000
$$$$
.000
$$$$
.0078125
$$$$
.0039063
$$$$
.0019531
$$$$
.0029297
$$$$
.0053711
$$$$
.0043945
$$$$
.0039063
$$$$
.0040894
$$$$
.0038147
$$

\section{Exact}

Answer

$$
0.0807455
$$

0.0064424

Error

0.0066

0.0026

Predicted

Error at

95\% Level
0.005900
0.000866

\section{Method II'}

Number of Points Sampled

$$
n=2
$$$$
n=9
$$

$\begin{array}{rr}32 & 0.8125000000 \\ 64 & .7500000000 \\ 128 & .7812500000 \\ 256 & .7695312500 \\ 512 & .7714843750 \\ 1024 & .7822265625 \\ 2048 & .7812500000 \\ 4096 & .7800292969 \\ 8192 & .7800292969 \\ 16384 & \\ 32768 & \end{array}$

0.0312500000

.0156250000

.0156250000

.0078125000

.0039062500

.0048828125

.0048828125

.0053710938

.0072021484

.0068359375

.0073852539

Exact

Answer

0.785398

0.006442

Error

0.005369

0.000943

Predicted

Error at

95\% Level

0.008890

0.000866 
amount of time required to compute $v_{n}$ is $2 n N \mu$, where $\mu$ is the multiplication time of the machine. The case $n=12, N=65,536$ consumed about $1 \frac{1}{2}$ hours on SEAC.

5. Some General Observations on Multiple Integration: One reason for carrying out the experiments described is this. A pseudo-random sequence is one which has passed (in some vague sense) a certain number of statistical tests. It is not clear $a$ priori that by combining its consecutive elements into $n$-tuples we obtain a pseudo-random sequence in $n$ dimensions. Therefore the above experiments constitute additional tests for the randomness of the sequence and, when successful, increase our confidence in its use in multiple integration.

For purposes of integration, it is not necessary to employ pseudo-random sequences; it suffices to employ sequences which are equidistributed [6]. Such sequences can be defined rigorously and the integration of sufficiently smooth functions can be carried out with a theoretical error of order $\mathrm{O}(1 / N)[7]$.

To obtain a picture of the difference in the speed of convergence, compare the final two tables. In the first of these tables, the integral $\int_{0}^{1} x d x$ was computed using the sequence (5). In the second table the same integral was computed using the sequence

$$
u_{j}=\left[j \frac{\sqrt{2}}{2}\right], \quad[x]=\text { fractional part of } x
$$

for which the theoretical error estimate is $\mathrm{O}(1 / N)$. The manner in which the former computation obeys the $1 / \sqrt{N}$ law, and the latter the $1 / N$ law is striking.

To integrate in $n$-dimensions, one makes use of the points $P_{j}:\left(\left[j \xi_{1}\right],\left[j \xi_{2}\right]\right.$, $\cdots,\left[j \xi_{n}\right]$ ) where $\xi_{i}$ are $n$ linearly independent irrational numbers belonging to some algebraic number field. The error estimate is again $\mathrm{O}(1 / N)$, but of course one must contend with a possible increase in the size of the constant which is implicit in the term $\mathrm{O}(1 / N)$.

Table III presents the results of an integration carried out in four dimensions using $\xi_{1}=\frac{\sqrt{2}}{2}, \xi_{2}=\sqrt{3}, \xi_{3}=\frac{\sqrt{6}}{3}, \xi_{4}=\sqrt{10}$. It would appear that these figures satisfy the $1 / N$ law although the associated constant is somewhat larger than that of the computation in Table II.

One final observation. The use of the word "Monte Carlo" or "sampling" in connection with quadratures camouflages the following computational fact of life: having agreed to invest in an $N$ point computation, we proceed to form $N$ fixed points $P_{1}, \cdots, P_{N}$ and use the approximation

$$
\int_{V} f(P) d v=V / N \sum_{j=1}^{N} f\left(P_{j}\right) .
$$

This is tantamount to the use of an $N$ point rule with abscissas $P_{j}$ and weights equal to $1 / N$. Such an $N$ point rule is incredibly inaccurate. For any fixed set of $N$ points, we can, in principle at least, select the weights so that the error is minimized in some sense [8]. Or we can select both abscissas and weights so that this error is minimized. It might prove fruitful to look into the possibility of obtaining best quadrature formulas of high order and in spaces of high dimension. 
TABLE II

No. of Points

$$
\begin{aligned}
& \text { Computation of } \int_{0}^{1} x d x \\
& \text { Computation of } \int_{0}^{1} x d x \\
& \text { Using the Sequence } u_{j} \\
& \text { Described by (5) } \\
& {\left[j \frac{\sqrt{2}}{2}\right]}
\end{aligned}
$$

Sampled

$$
\begin{aligned}
& \begin{array}{ll}
0.3703920880 & 0.5606601718
\end{array} \\
& .5216710794 \quad .5177669530 \\
& .5064453536 \quad .5569805153 \\
& .4547871881 \quad \mathbf{5 1 0 4 0 7 6 4 0 1} \\
& .5015472909 \quad \mathbf{5 1 1 0 1 1 8 8 9 6} \\
& .5204149952 \quad .4965953886 \\
& .5268724155 \quad .4990123865 \\
& .5070362771 \quad .4999401325 \\
& .5122595896 \quad .4998424994 \\
& .5147580545 \quad .4996472331 \\
& .5140853905 \quad .4997449819 \\
& .5083722340 \quad .4999404795 \\
& .5031562483 \quad .5000873340 \\
& .5026730074 \quad .5000148320 \\
& .5019506662 \quad .5000224160 \\
& .5028558372 \quad .5000070665 \\
& .5016144215 \quad .4999992555 \\
& \begin{array}{ll}
.5008443891 & .4999988924
\end{array} \\
& \text { Computation of } \int_{0}^{1} \int_{0}^{1} \int_{0}^{1} \int_{0}^{1} e^{x_{1} x_{2} x_{3} x_{4}} d x_{1} d x_{2} d x_{3} d x_{4} \text { Using the Sequence } \\
& \left(\left[j \frac{\sqrt{2}}{2}\right],[j \sqrt{3}],\left[j \frac{\sqrt{6}}{3}\right],[j \sqrt{10}]\right)
\end{aligned}
$$

$\begin{array}{rl}2 & 1.0556385354 \\ 4 & 1.0646192182 \\ 8 & 1.0592766300 \\ 16 & 1.0615566540 \\ 32 & 1.0626118843 \\ 64 & 1.0586261203 \\ 128 & 1.0657314237 \\ 256 & 1.0673118827 \\ 512 & 1.0668402647 \\ 1024 & 1.0681499894 \\ 2048 & 1.0685418201 \\ 4096 & 1.0685544568 \\ 8192 & 1.0688021121 \\ 16384 & 1.0691568057 \\ 32768 & 1.0691964247 \\ \text { Exact Answer } & 1.06939761\end{array}$

National Bureau of Standards

Washington, D. C.

Weitzmann Institute

Rehoboth, Israel 
This work was supported in part by the Office of Naval Research.

1. See, however: J. ToDd, "Experiments in the solution of differential equations by Monte Carlo methods," J. Wash. Acad. of Sciences, v. 44, 1954, p. 377-381; N. Metropolis, A. W. Rosenbluth, M. N. Rosenbluth, A. H. Teller, \& E. Teller, "Equation of state calculations by fast computing machines," J. Chem. Phys., v. 21, 1953, p. 1087-1092; R. D. RICHTMYER, "The evaluation of definite integrals and a quasi-Monte Carlo method based on the properties of algebraic numbers," Los Alamos Rep. 13, Oct. 1951.

2. H. KaHN, "Modification of the Monte Carlo method," Seminar on Scientific Computation, International Business Machines Corp., November 1949.

3. For more detail on these methods see: O. TAUsSKY \& J. TodD, "The generation and testing of pseudo-random numbers on SEAC." To appear.

4. Ibid.

5. See, e.g., H. KaHN, loc. cit.

6. See, e.g., G. Pólya \& G. Szegö, Aufgaben und Lehrsätze aus der Analysis, Dover Publications, Inc., New York, v. 1, 1945, p. 70-77.

7. RICHTMYER, loc. cit.

8. ARTHUR SARD, "Best approximate integration formulas," Am. J. Math., v. 71, 1949, p. 80-91; S. M. NikolskiI, "K voprosa ob otzenkakh priblizhenii kvadraturnimi formulami," Uspekhi Math. Nauk, v. 51, p. 165-177; Philip DAVIS, "Errors of numerical approximation for analytic functions," J. Rat. Mech. and Anal., v. 2, 1953, p. 303-313.

\section{Generating and Testing Pseudo Random Numbers on the IBM Type 701}

1. Introduction. With the increased use of large-scale digital computing machines many problems too complicated to solve analytically, or even numerically, have been solved by the so-called Monte Carlo method. The Monte Carlo technique employs repeated sampling to evaluate integrals or to simulate physical problems directly and requires a large supply of random numbers distributed in a manner resembling the true distribution of the physical quantities which they represent.

A set of random numbers of any specified distribution can be, and usually is, obtained from a set of random numbers uniformly distributed on the interval 0 to 1 . If we are given the density function $f(x)$ of the desired distribution and can integrate this density function in closed form, the most direct way to get the desired set of random numbers $\left\{N_{i}\right\}$ from a set of uniformly distributed random numbers, $\left\{n_{i}\right\}$, is to solve for $N_{i}$ in the equation

$$
n_{i}=\int_{-\infty}^{N_{i}} f(x) d x .
$$

However, the number of cases where the integral can be evaluated and the resulting equation solved explicitly for $N_{i}$ is small, especially when such solution must be simple enough to be repeated the large number of times required for an extensive table. In some cases the density function $f(x)$ may be approximated by some simpler function, a polynomial say, to afford an approximation of (1) which can easily be solved for $N_{i}$.

In some other cases there are special properties of the distribution of $N_{i}$ by which one is able to get the new set of numbers quite directly from the original set. One example is the normal distribution, or error function, a common distribution of random quantities encountered in physical problems. From the central limit theorem of probability we know that sums of uniformly distributed random variables will approach the normal distribution as the number of random variables 